\title{
Usefulness of Goal Attainment Scaling in Intensive Stroke Rehabilitation During the Subacute Stage
}

\author{
Youngsu Jung, MD, Jaehoon Sim, MD, Joonhyun Park, MD, Jongmoon Kim, MD, MinYoung Kim, MD, PhD \\ Department of Rehabilitation Medicine, CHA Bundang Medical Center, CHA University College of Medicine, Seongnam, Korea
}

Objective To investigate the usefulness of goal attainment scaling (GAS) in intensive stroke rehabilitation during the subacute stage.

Methods Medical records of subacute post-stroke patients who had undergone intensive rehabilitation under hospitalization, before and after the application of GAS, were collected. GAS was conducted at the initial evaluation of each patient. Specific goals were suggested by physical and occupational therapists and were determined by the responsible physiatrist. A 5-point scale was used for the GAS score after 4 weeks of rehabilitation according to the preset criteria of each goal. To evaluate the influence of GAS in rehabilitation practice, functional improvements were compared between two patient groups before $(n=121)$ and after $(n=141)$ GAS. To assess progress in GAS practice, the standard GAS score was calculated, and the changes were observed over a 3-year period. The standard GAS score converged to 50 points when the goal was achieved. The therapists who used GAS also completed a survey regarding its usefulness.

Results There were no statistical differences in the motor and cognitive outcomes of the patient groups before and after applying GAS scoring. Successive yearly changes in the standard GAS scores showed progressive convergence to 50 points, signaling that the patient's goal-setting abilities improved. According to the survey, most therapists felt that GAS enhanced the quality of therapies (84.6\%).

Conclusion GAS improved goal-setting for the rehabilitation of subacute post-stroke patients and might have a positive effect on rehabilitation.

Keywords Stroke, Rehabilitation, Physical therapy, Occupational therapy, Goals

Department of Rehabilitation Medicine, CHA Bundang Medical Center, CHA University College of Medicine, 59 Yatap-ro, Bundang-gu, Seongnam 13496, Korea. Tel: +82-31-780-1872, Fax: +82-31-780-3449, E-mail: kmin@cha.ac.kr

ORCID: Youngsu Jung (http://orcid.org/0000-0001-8173-736X); Jaehoon Sim (http://orcid.org/0000-0003-2276-1743); Joonhyun Park (http://orcid. org/0000-0002-6782-670X); Jongmoon Kim (http://orcid.org/0000-0002-8684-8736); MinYoung Kim (http://orcid.org/0000-0001-5481-2985).

(c) This is an open-access article distributed under the terms of the Creative Commons Attribution Non-Commercial License (http://creativecommons.org/ licenses/by-nc/4.0) which permits unrestricted noncommercial use, distribution, and reproduction in any medium, provided the original work is properly cited. Copyright ( 2020 by Korean Academy of Rehabilitation Medicine 


\section{INTRODUCTION}

Stroke is the most common cause of acquired disability [1]. The impairments caused by stroke can vary $[2,3]$. Rehabilitation therapy can help restore the function of stroke-caused impairments [4]. Rehabilitative interventions work best when the patient's medical, neurological, and psycho-social conditions are holistically considered [5]. From a practical perspective, precise goal setting is an important element of rehabilitative therapy [6]. However, given the variable stroke-induced neurologic impairment status and personal medical conditions (age, stage after the stroke onset), it is difficult to set precise goals when implementing a rehabilitation program [7].

Goal attainment scaling (GAS) was first introduced in the 1960s as a patient-specific methodology based on the patient's functional status and goals $[8,9]$. GAS aims to establish realistic expectations by consulting with the patient and their family before the intervention. Achievement is evaluated on a 5- or 6-point scale [10]. GAS has a number of benefits including (1) setting priorities in establishing rehabilitation programs, (2) establishing team meetings and multidisciplinary consultations with clear objectives, (3) quantifying the patient's progress, and (4) involving the patient as well as the patient's family in the treatment process to ensure that the goal is established and evaluated $[11,12]$. Also, as patients have a key role in setting their goals, they may be more motivated in the treatment process [7].

Due to the advantages mentioned above, GAS has been implemented in clinical practice to set and evaluate goals in a variety of areas such as elderly care, cognitive rehabilitation, and amputee rehabilitation [10]. There have been several reports on the use of GAS in the rehabilitation of patients with brain injuries [13,14]. However, there is a lack of studies that examine the use of GAS in intensive rehabilitation during the subacute phase of stroke [4]. The subacute phase presents the biggest opportunity for recovery.

Our clinical study team based at a rehabilitation center in a university hospital has used GAS since 2016 in poststroke rehabilitation therapy during the subacute stage based on decades of experience. After it was applied for 3 years, the study team evaluated the validity of GAS used in intensive stroke rehabilitation at the subacute stage. First, the functional outcomes before and after the ap- plication of GAS were compared. Second, yearly changes in goal setting patterns were analyzed using the standard GAS score [11], which assessed the attainment levels of multiple goals over the 3-year period. The standard GAS score was converted to 50 points when the goal was achieved as expected. The closer the score was to 50 , the better the quality of the goal [10]. Third, we analyzed factors that might affect the standard GAS score. Finally, the therapists who used GAS completed a survey regarding its usefulness.

\section{MATERIALS AND METHODS}

\section{Subjects}

This study was approved by the Institutional Review Board of CHA Bundang Medical Center (No. 2019-03009) and the informed consent was waived.

Medical records of hospitalized patients who were over 18 years old during the period from January 2014 to December 2018 were obtained for patients that had undergone intensive rehabilitation in the subacute poststroke stage. The inclusion criteria were patients who undertook functional evaluations at the time of admission and after 1 month of rehabilitation. In this study, the subacute stage was defined as stroke onset 15 to 90 days before admission or transfer into the rehabilitation medicine department $[15,16]$. The exclusion criteria were patients who were unable to undertake the Mini-Mental State Examination (MMSE) or had a score of 0 and a preexisting disability, which might affect activities of daily living (ADL).

\section{Outcome measures}

The study site specializes in the rehabilitation of poststroke patients, especially during the subacute stage. At the initiation of intensive rehabilitation, the patients had their gross motor ability of strength and mobility routinely evaluated using the manual muscle test (MMT) by the Medical Research Council score, Berg Balance Scale (BBS), Trunk Impairment Scale (TIS), and Functional Ambulatory Category (FAC), which were the domain of physical therapy (PT). The patients cognition, perception, upper limb function and ADL were also evaluated, using the MMSE, Motor-Free Visual Perception Test (MVPT-3), Fugl-Meyer Assessment (FMA) for the upper extremities, Manual Function Test (MFT), Functional Independence 
Measure (FIM), and Modified Barthel Index (MBI), which were the domain of occupational therapy (OT). These evaluations were conducted before the initiation of rehabilitation and were repeated after 4 weeks of rehabilitation. The members of the clinical research team assessed inter-rater reliabilities for the functional evaluation items annually to establish the competence of the rehabilitation processes and the results of last year as described in Supplementary Table S1, which indicates appropriate reliabilities for clinical use in the university hospital (intraclass correlation coefficient $>0.90, \mathrm{p}<0.001$ ).

To examine changes in the rehabilitative outcomes after using GAS, clinical data of functional measures from January 2014 to December 2015, the period before application of GAS, and the same data from January 2016 to December 2018, the period after application of GAS, were compared.

\section{GAS and the standard GAS score}

In this rehabilitation center, GAS has been used for all brain-damaged patients since 2016. Medical staff and therapists treat patients according to the goals which are set when rehabilitation is initiated. Three specific goals are selected in each of the domains, PT and OT. In the PT domain, three goals are selected from the following categories: functions of the joints and bones (b710-b729), muscle functions (b730-b749), movement functions (b750-b789), changing and maintaining body position (d410-d429), walking and moving (d450-d469), and community, social, and civic life (d910-d999) according to the International Classification of Functioning, Disability, and Health (ICF) [17]. In the OT domain, three goals were selected from the following categories: specific mental functions (b140-b189), carrying, moving and handling objects (d430-d449), self-care (d510-d599), household tasks (d630-d649), work and employment (d840-d859), and community, social and civic life (d910-d999) according to the ICF. Multiple goals could be set within one category. Goals that could be assessed as evaluation items such as MMT, BBS, TIS, and FAC were expressed as scores. Otherwise, they were expressed in clear sentences, such as "You can walk the treadmill for 20 minutes at a speed of $5.0 \mathrm{~km} / \mathrm{hr}$ and a 5 -degree slope" in the PT domain. In the OT domain, goals that could be evaluated as evaluation items such as FIM or MBI, FMA or MFT, and K-MMSE or MVPT-3 were expressed as scores. Otherwise, they were expressed in clear sentences, such as "You can iron your t-shirt without wrinkles". Concrete goals were suggested by therapists and were determined after a team meeting with the corresponding physiatrist after functional evaluations and discussions with the patient or their caregiver. In the team meeting, appropriate goals were selected after a discussion about the patient's medical status, neurological impairments, the extent of brain injury, electrophysiological study, age, sex, and expected role at home or in society. Moreover, for patients who completed 4 weeks of rehabilitation, GAS scores were assessed in the team meeting, and feedback on whether the goal setting was appropriate was discussed.

GAS was scored on a 5-point scale: the center scale of 0 indicated the expected level of achievement; +1 , higher than expected; +2 , far higher than expected; -1 , lower than expected but still better than baseline function; -2 , decreased function than baseline status.

To comprehensively evaluate the achievement of the goals set in the PT and OT domains, we used the "standard GAS score". This has been used by most researchers that have focused on GAS [8]. The formula for obtaining this standard score is as follows:

Standard GAS score

$$
\left.=50+\left\{\left(10 \Sigma\left(w_{i} X_{i}\right)\right) /(1-\rho) \Sigma W_{i}^{2}+\rho\left(\Sigma W_{i}\right)^{2}\right)^{1 / 2}\right\} .
$$

where $w_{i}$ is the assigned weight to the ith goal (if weights are equal, $\left.w_{i}=1\right) ; x_{i}$ is the numerical GAS value of each goal (between -2 and +2 ); and $\rho$ is the expected correlation of the goal scales.

For practical purposes, $\rho$ commonly approximates to 0.3 [8], so the equation simplifies to:

Standard GAS score

$$
=50+\left\{\left(10 \Sigma\left(W_{i} X_{i}\right)\right) /\left(0.7 \Sigma W_{i}^{2}+0.3\left(\sum W_{i}\right)^{2}\right)^{1 / 2}\right\} .
$$

The standard GAS score is a converted score on a standardized scale, i.e., T-score, with an average value is 50 and a standard deviation of 10 . This standard GAS score is the value for the achievement degree of the overall goals. If all the goals have been properly setup according to the formula above (all $x_{\mathrm{i}} \mathrm{s}=0$ ), the standard GAS score will be 50 points [10]. Therefore, the standard GAS score can be an indicator of the quality of GAS. If the team sets the goal too low, the standard GAS score will exceed 50 points. Conversely, if the team sets goals too high, the standard GAS score will be less than 50 points. In this study, all assigned weights $\left(w_{i}\right)$ for the three selected items in each domain were treated the same. 
The yearly standard GAS scores for the study population were assessed for the PT and OT domains over 3 years. This was done to see whether experience changed the quality of goal setting.

By analyzing the clinical data, we wanted to identify the factors that may affect the standard GAS score, such as age, post-stroke duration, stroke recurrence, stroke location (cerebral hemisphere and brainstem or cerebellum), laterality of stroke (right, left, and both), and initial functional status. Besides, we compared the baseline characteristics and results of the GAS scores between those with poor cognition (MMSE <20) and better cognition (MMSE $\geq 20$ ) to determine the impact of cognitive status on the GAS score [18].

\section{Questionnaires for therapists}

In the clinical study team, there were seven physical therapists and six occupational therapists. The average duration that they had worked in the team was 6.7 years (range, 2-21 years) for physical therapists and 9.2 years (range, 2-20 years) for occupational therapists. The therapists were given a survey to assess the practical usefulness of GAS, which included the following: (1) GAS is a good way to evaluate treatment results; (2) application of GAS facilitated setting specific goals, (3) use of GAS improves the quality of the rehabilitation treatment; (4) by using GAS, patients can participate with a good understanding of their treatment process; and (5) use of GAS enhances the motivation of the patient.

These items were evaluated on a 5-point Likert scale [19] as strongly agree, agree, or both agree nor disagree, disagree, and strongly disagree.

\section{Data analysis}

To analyze the influence in rehabilitation practice, functional outcomes from 2014 and 2015 were used as a historical control and compared with those after GAS was established at our institution in January 2016.

The following statistical analyses were used to compare the differences in baseline characteristics before and after the introduction of GAS. Age and post-stroke duration were compared using the independent t-test, and normality was verified using the Kolmogorov-Smirnov test. Evaluation scores were compared by the Mann-Whitney U-test. Categorical data were compared by the chi-square test. The paired t-test was used to compare the evalua- tion results before and after rehabilitation from January 2014 to December 2015 (the period before application of GAS) and from January 2016 to December 2018 (the period after application of GAS), respectively. The independent t-test was used to compare the degrees of changes in evaluations before and after the application of GAS, and normality was verified using the Kolmogorov-Smirnov test. A logistic regression analysis was used to evaluate the effect of variables such as age, post-stroke duration, stroke recurrence, stroke location (cerebral hemisphere and brainstem or cerebellum), laterality of stroke (right, left, and bilateral), and initial functional status after stroke (evaluated with MBI) on the achievement of a goal (T-score $\geq 50$ ). For making comparisons between groups according to the MMSE scores, categorical data were compared using the chi-square test and other characteristics using the Mann-Whitney U-test. The Statistical Package for the Social Sciences version 21.0 for Windows (IBM SPSS, Armonk, NY, USA) was used for the data analysis.

\section{RESULTS}

\section{Patient characteristics}

The clinical data of 262 subacute phase stroke patients who were admitted to the study hospital for rehabilitation from 2014 to 2018 were used. One hundred twenty-one patients seen in 2014-2015 (before GAS was introduced), and 141 patients seen in 2016-2018 (after GAS was introduced) were enrolled in the study. The mean age of the subjects was $61.2 \pm 14.8$ years ( $58.7 \%$ male) in 2014-2015, and $60.3 \pm 13.8$ years $(58.2 \%$ male) in $2016-2018$. An initial functional assessment was performed $38.2 \pm 27$.1 days after the onset of stroke in 2014-2015 and 34.5 \pm 22.4 days in subjects seen in 2016-2018. There were no differences in the baseline characteristics of the subjects between the two time periods regarding age, sex distribution, poststroke duration at the initial assessment, type of stroke, and initial functional assessment scores (Table 1).

\section{Effect on rehabilitation outcomes}

There were no significant differences in functional improvements by rehabilitation treatment before the introduction of GAS (2014 and 2015) as a historical control and after introducing GAS (2016, 2017, and 2018), while both groups showed significant improvement in most of 
Table 1. Demographic characteristics of the patients

\begin{tabular}{|lcc|}
\hline & $\mathbf{2 0 1 4 - 2 0 1 5}(\mathbf{n}=\mathbf{1 2 1})$ & $\mathbf{2 0 1 6 - 2 0 1 8}(\mathbf{n}=\mathbf{1 4 1})$ \\
\hline Age (yr) & $61.2 \pm 14.8$ & $60.3 \pm 13.8$ \\
\hline Sex & & \\
\hline Male & 71 & 58 \\
\hline Female & 50 & $60(42.6)$ \\
\hline Etiology & $54(44.6)$ & $81(57.4)$ \\
\hline Cerebral infarction & $67(55.4)$ & \\
\hline Intracerebral hemorrhage & & $69(48.9)$ \\
\hline Lesion & $60(49.6)$ & $54(38.3)$ \\
\hline Right hemisphere & $46(38.0)$ & $8(5.7)$ \\
\hline Left hemisphere & $7(5.8)$ & $2(1.4)$ \\
\hline Bilateral hemispheres & $1(0.8)$ & $8(5.7)$ \\
\hline Cerebellum & $7(5.8)$ & $34.5 \pm 22.4$ \\
\hline Brainstem & $38.2 \pm 27.1$ & $3(2.1)$ \\
\hline Post-stroke duration (day) & $3(2.5)$ & $15.6 \pm 8.6$ \\
\hline Recurred stroke & $15.2 \pm 8.9$ & $31.0 \pm 24.1$ \\
\hline MMSE score & $30.8 \pm 25.2$ & $36.3 \pm 34.1$ \\
\hline FMA score & $35.3 \pm 35.2$ & $35.9 \pm 23.8$ \\
\hline MFT score & $34.2 \pm 23.9$ & $18.8 \pm 8.4$ \\
\hline MBI score & & $36.5 \pm 17.4$ \\
\hline FIM score & $16.9 \pm 8.6$ & $12.2 \pm 5.3$ \\
\hline Cognition (n=58) & $32.4 \pm 15.6$ & $17.7 \pm 18.2$ \\
\hline Motor (n=58) & $12.0 \pm 6.1$ & $8.35 \pm 6.61$ \\
\hline MMT score & $16.7 \pm 17.8$ & $1.27 \pm 1.27$ \\
\hline BBS score & $8.22 \pm 6.02$ & $1.20 \pm 1.27$ \\
\hline TIS score & & \\
\hline FAC & & \\
\hline
\end{tabular}

Values are presented as mean \pm standard deviation or number (\%).

The study hospital began the Wee-FIM assessment from 2015 and included only the results for 58 patients. The sum of the manual muscle test by Medical Research Council score was divided into 6 muscle groups including shoulder abductor of the weaker side, elbow flexor, wrist extensor, hip flexor, knee extensor, and ankle dorsiflexor. It ranges from 0 (totally hemiplegic) to 30 (normal strength).

MMSE, Mini-Mental State Examination; FMA, Fugl-Meyer Assessment; MFT, Manual Function Test; MBI, Modified Barthel Index; FIM, Functional Independence Measurement; MMT, Manual Muscle Test; BBS, Berg Balance Scale; TIS, Trunk Impairment Scale; FAC, Functional Ambulatory Category.

Age and post-stroke duration were compared by independent t-test. Evaluation scores were compared by Mann-Whitney U-test. Number of each group was compared by chi-square test. There was no significant difference in baseline characteristics of patients during both periods.

the evaluated function measures (Table 2).

\section{Quality check for goal setting}

When the mean of the standard GAS score for the three years of 2016, 2017, and 2018 was arranged in order of time, the standard GAS score gradually moved towards 50 points in both the PT and OT domains (Fig. 1). In the PT, the standard GAS scores were $53.4 \pm 7.9$ in $2016,52.7 \pm 7.1$ in 2017 , and $50.5 \pm 7.3$ in 2018. In the OT, the standard GAS scores were $46.8 \pm 7.7$ in $2016,47.5 \pm 6.8$ in 2017 , and $48.9 \pm 4.9$ in 2018. It seems that the goals of the PT domain tended to be set low, and the goals of the OT domain tended to be set high. However, the goals became increasingly more appropriate with experience. 
Table 2. Comparison of rehabilitation outcomes before and after introduction of goal attainment scaling

\begin{tabular}{|c|c|c|c|c|c|c|c|c|c|}
\hline \multirow[b]{2}{*}{$\begin{array}{l}\text { Assessments } \\
\text { (range) }\end{array}$} & \multicolumn{4}{|c|}{$2014-2015(n=121)$} & \multicolumn{4}{|c|}{$2016-2018(n=141)$} & \multirow[b]{2}{*}{$\begin{array}{c}\text { p-value } \\
\text { (between } \\
\text { groups) }\end{array}$} \\
\hline & Baseline & Follow-up & Change & $\begin{array}{l}\text { p-value } \\
\text { (within } \\
\text { groups) }\end{array}$ & Baseline & Follow-up & Change & $\begin{array}{l}\text { p-value } \\
\text { (within } \\
\text { groups) }\end{array}$ & \\
\hline $\begin{array}{l}\text { MMSE score } \\
(0-30)\end{array}$ & $15.2 \pm 8.9$ & $21.1 \pm 8.2$ & $5.9 \pm 5.1$ & $<0.01$ & $15.6 \pm 8.6$ & $21.2 \pm 7.9$ & $5.6 \pm 4.4$ & $<0.01$ & 0.55 \\
\hline $\begin{array}{l}\text { FMA score } \\
(0-66)\end{array}$ & $30.8 \pm 25.2$ & $38.4 \pm 25.3$ & $7.6 \pm 11.1$ & $<0.01$ & $31.0 \pm 24.1$ & $38.3 \pm 25.4$ & $7.3 \pm 10.3$ & $<0.01$ & 0.63 \\
\hline $\begin{array}{l}\text { MFT score } \\
(0-100)\end{array}$ & $35.3 \pm 35.2$ & $47.7 \pm 37.2$ & $12.4 \pm 13.5$ & $<0.01$ & $36.3 \pm 34.1$ & $49.1 \pm 38.1$ & $12.8 \pm 15.3$ & $<0.01$ & 0.84 \\
\hline $\begin{array}{l}\text { MBI score } \\
(0-100)\end{array}$ & $34.2 \pm 23.9$ & $53.5 \pm 27.2$ & $19.3 \pm 13.3$ & $<0.01$ & $35.9 \pm 23.8$ & $55.2 \pm 27.6$ & $19.3 \pm 13.4$ & $<0.01$ & 0.73 \\
\hline \multicolumn{10}{|l|}{ FIM score } \\
\hline $\begin{array}{l}\text { Cognition } \\
(0-35)\end{array}$ & $16.9 \pm 8.6^{\mathrm{a})}$ & $20.9 \pm 9.0^{\mathrm{a})}$ & $4.0 \pm 4.0^{\mathrm{a})}$ & $<0.01$ & $18.8 \pm 8.4$ & $22.5 \pm 8.3$ & $3.7 \pm 3.7$ & $<0.01$ & 0.54 \\
\hline $\begin{array}{l}\text { Motor } \\
(0-91)\end{array}$ & $32.4 \pm 15.6^{\mathrm{a})}$ & $46.2 \pm 22.9^{\mathrm{a})}$ & $13.8 \pm 10.6^{\mathrm{a})}$ & $<0.01$ & $36.5 \pm 17.4$ & $50.5 \pm 21.1$ & $14.0 \pm 10.2$ & $<0.01$ & 0.40 \\
\hline $\begin{array}{l}\text { MMT score } \\
\quad(0-30)\end{array}$ & $12.0 \pm 6.1$ & $14.4 \pm 6.1$ & $2.4 \pm 2.8$ & $<0.01$ & $12.2 \pm 5.3$ & $14.6 \pm 5.8$ & $2.4 \pm 2.5$ & $<0.01$ & 0.39 \\
\hline $\begin{array}{c}\text { BBS score } \\
(0-56)\end{array}$ & $16.7 \pm 17.8$ & $30.3 \pm 20.4$ & $13.6 \pm 12.8$ & $<0.01$ & $17.7 \pm 18.2$ & $31.4 \pm 20.5$ & $13.7 \pm 11.6$ & $<0.01$ & 0.82 \\
\hline $\begin{array}{l}\text { TIS score } \\
(0-23)\end{array}$ & $8.22 \pm 6.02$ & $12.47 \pm 6.32$ & $4.25 \pm 3.56$ & $<0.01$ & $8.35 \pm 6.61$ & $12.84 \pm 6.77$ & $4.49 \pm 3.82$ & $<0.01$ & 0.48 \\
\hline $\begin{array}{l}\text { FAC } \\
(0-5) \\
\end{array}$ & $1.20 \pm 1.27$ & $2.31 \pm 1.57$ & $1.11 \pm 0.92$ & $<0.01$ & $1.27 \pm 1.27$ & $2.42 \pm 1.60$ & $1.15 \pm 0.94$ & $<0.01$ & 0.65 \\
\hline
\end{tabular}

Values are presented as mean \pm standard deviation.

The study hospital began the Wee-FIM assessment from 2015 and included only the results for 58 patients. The sum of the manual muscle test by Medical Research Council score was divided into 6 muscle groups including shoulder abductor of the weaker side, elbow flexor, wrist extensor, hip flexor, knee extensor, and ankle dorsiflexor. It ranges from 0 (totally hemiplegic) to 30 (normal strength).

MMSE, Mini-Mental State Examination; FMA, Fugl-Meyer Assessment; MFT, Manual Function Test; MBI, Modified Barthel Index; FIM, Functional Independence Measurement; MMT, Manual Muscle Test; BBS, Berg Balance Scale; TIS, Trunk Impairment Scale; FAC, Functional Ambulatory Category.

Each score of baseline and follow-up within same group was compared by paired t-test. The independent t-test was used to compare changes in each score (score at follow-up minus score at admission) between both periods. There was no significant difference in all assessments between both periods.

${ }^{a)} \mathrm{n}=58$.

\section{GAS scores}

The rehabilitation goals are listed in Table 3, according to the main chapters of ICF in the PT and OT domains, respectively. The categories in which function was higher than the goal (GAS $\geq 0$ ) in the PT domain were: functions of the joints and bones (b710-b729; 68.0\%), muscle function (b730-b749; 86.5\%), movement functions (b750b789; 76.5\%), changing and maintaining body position (d410-d429; 75.0\%), walking and moving (d450-d469; 83.5\%), and community, social, and civic life (d910-d999;
88.0\%). The categories in which function was higher than the goal (GAS $\geq 0$ ) in the OT domain were: specific mental function (b140-b189; 73.9\%), carrying, moving and handling objects (d430-d449; 54.6\%), self-care (d510-d599; $78.3 \%$ ), household tasks (d630-d649; 75.0\%), work and employment (d840-d859; 80.0\%), and community, social and civic life (d910-d999; 75.0\%).

\section{Factors affecting the standard GAS score}

According to the logistic regression, post-stroke dura- 

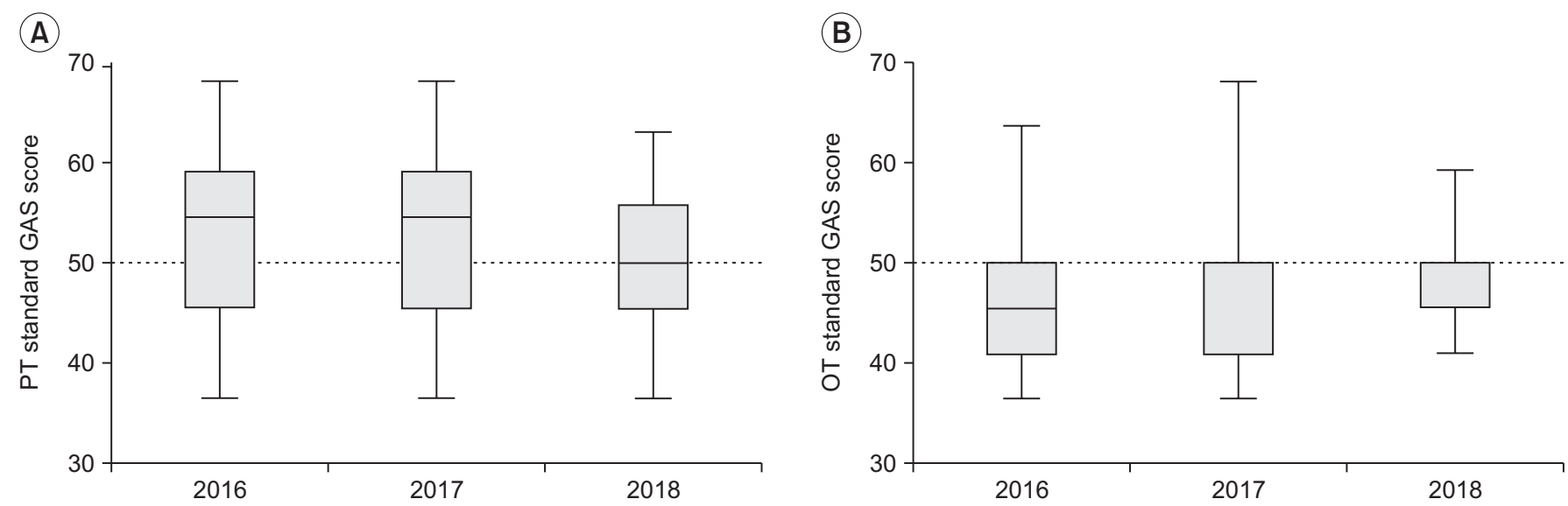

Fig. 1. Changes in standard goal attainment scaling (GAS) scores by year. (A) Physical therapy (PT) and (B) occupational therapy (OT). The box-and-whiskers plots represent line is median value; box, upper and lower quartiles; whiskers, 5\%-95\% confidence. The median values are displayed above the box.

Table 3. Classification of goal domain and distribution of GAS scores

\begin{tabular}{|c|c|c|c|c|c|c|c|c|}
\hline \multirow[t]{2}{*}{ Patient goals according to ICF } & \multirow{2}{*}{$\begin{array}{l}\text { \% of patients } \\
\text { with goal }\end{array}$} & \multicolumn{4}{|c|}{$\begin{array}{c}\text { Number of patients } \\
\text { achieved or exceeded the goal }\end{array}$} & \multicolumn{3}{|c|}{$\begin{array}{l}\text { Number of patients } \\
\text { did not meet the goal }\end{array}$} \\
\hline & & $\mathbf{0}$ & +1 & +2 & Total & -1 & -2 & Total \\
\hline \multicolumn{9}{|l|}{ Physical therapy } \\
\hline $\begin{array}{l}\text { Functions of the joints and bones } \\
\text { (b710-b729) }\end{array}$ & 68.0 & 37 & 13 & 1 & 51 & 24 & 0 & 24 \\
\hline Muscle functions (b730-b749) & 86.5 & 46 & 18 & 0 & 64 & 8 & 2 & 10 \\
\hline Movement functions (b750-b789) & 76.5 & 48 & 14 & 3 & 65 & 20 & 0 & 20 \\
\hline $\begin{array}{l}\text { Changing and maintaining body } \\
\text { position (d410-d429) }\end{array}$ & 75.0 & 24 & 7 & 2 & 33 & 11 & 0 & 11 \\
\hline Walking and moving (d450-d469) & 83.5 & 66 & 27 & 8 & 101 & 20 & 0 & 20 \\
\hline $\begin{array}{l}\text { Community, social and civic life } \\
\text { (d910-d999) }\end{array}$ & 88.0 & 13 & 8 & 1 & 22 & 3 & 0 & 3 \\
\hline \multicolumn{9}{|l|}{ Occupational therapy } \\
\hline $\begin{array}{l}\text { Specific mental functions } \\
\text { (b140-b189) }\end{array}$ & 73.9 & 65 & 16 & 1 & 82 & 29 & 0 & 29 \\
\hline $\begin{array}{l}\text { Carrying, moving and handling } \\
\text { objects (d430-d449) }\end{array}$ & 54.6 & 45 & 13 & 1 & 59 & 48 & 1 & 49 \\
\hline Self-care (d510-d599) & 78.3 & 101 & 15 & 3 & 119 & 32 & 1 & 33 \\
\hline Household tasks (d630-d649) & 75.0 & 8 & 1 & 0 & 9 & 3 & 0 & 3 \\
\hline $\begin{array}{l}\text { Work and employment } \\
\text { (d840-d859) }\end{array}$ & 80.0 & 16 & 4 & 0 & 20 & 5 & 0 & 5 \\
\hline $\begin{array}{l}\text { Community, social and civic life } \\
\text { (d910-d999) }\end{array}$ & 75.0 & 8 & 4 & 0 & 12 & 4 & 0 & 4 \\
\hline
\end{tabular}

GAS, goal attainment scaling; ICF, International Classification of Functioning, Disability and Health.

tion was found to have a minor but negative effect on goal attainment in the PT domain. As post-stroke duration at the initial evaluation increased by one day, the likelihood of achieving the goal in the PT was reduced to 0.979 times. Other factors such as stroke recurrence, stroke location (cerebral hemisphere and brainstem or 
cerebellum), laterality of stroke (right, left, and bilateral), and initial function after stroke (evaluated with MBI) did not significantly affect goal achievement in the PT domain. In the OT domain, all factors, including post-stroke duration, did not significantly affect goal achievement (Table 4).

The GAS score was significantly higher in the group with an MMSE score $\geq 20$ than the group with an MMSE score $<20$ in both the PT and OT domains. However, there were also significant differences between the two groups in other characteristics such as age, location of the lesions, post-stroke duration, and all evaluation scores (MMSE, FMA, MFT, MBI, FIM, MMT, BBS, TIS, and FAC) (Table 5).

Fig. 2 shows the change in the mean standard GAS scores in the PT and OT domains over the 3 years in the group with an MMSE score $<20$ and the group with an MMSE score $\geq 20$. In the group with an MMSE $<20$, the standard GAS scores were $50.6 \pm 7.6$ in $2016,50.2 \pm 8.3$ in 2017, and 48.6 \pm 8.0 in 2018 in the PT domain, and $47.3 \pm 8.0$ in 2016, 45.8 \pm 6.4 in 2017, and 48.4 \pm 5.4 in 2018 in the OT domain. In the group with an MMSE score $\geq 20$, the standard GAS scores were $56.5 \pm 6.9$ in $2016,55.4 \pm 7.4$ in 2017, and 52.6 \pm 6.0 in 2018 in the PT domain, and $46.4 \pm 7.8$ in 2016, $51.0 \pm 6.4$ in 2017, and $49.5 \pm 4.3$ in 2018 in the OT domain. There were no significant differences in the distribution of the number of patients by MMSE score and post-stroke duration between the different time periods (Supplementary Table S2).

\section{Questionnaires for therapists}

Most of the therapists agreed that "GAS is a good way to evaluate treatment results (84.6\%)", "Application of GAS facilitated setting specific goals (84.6\%)", "Use of GAS improves the quality of the rehabilitation treatment (84.6\%)", "By using GAS, patients can participate with a good understanding of their treatment process (76.9\%)", and "Use of GAS enhances motivation of the patient (69.2\%)". With

Table 4. Result of logistic regression on goal attainment as a dependent variable

\begin{tabular}{|lccccc}
\hline \multirow{2}{*}{ Independent variable } & \multicolumn{5}{c}{ Dependent variable } \\
\cline { 2 - 5 } & $\mathbf{B}$ & SE & p-value & OR & $\mathbf{9 5 \%}$ CI \\
\hline Physical therapy & & & & & \\
$\quad$ Age & -0.027 & 0.019 & 0.167 & 0.974 & 0.937 to 1.011 \\
\hline Post-stroke duration (day) & -0.021 & 0.010 & $0.032^{*}$ & 0.979 & 0.959 to 0.998 \\
\hline Stroke etiology & -0.160 & 0.535 & 0.765 & 0.852 & -0.197 to 1.900 \\
\hline Stroke recurrence & -0.409 & 1.336 & 0.759 & 0.664 & -1.955 to 3.283 \\
\hline Laterality & 0.274 & 0.451 & 0.543 & 1.315 & 0.431 to 2.199 \\
Location & 19.916 & $1.213 \times 10^{4}$ & 0.999 & $4.461 \times 10^{8}$ & $-4.461 \times 10^{8}$ to $4.461 \times 10^{8}$ \\
\hline MBI & 0.016 & 1.757 & 0.185 & 1.016 & -2.428 to 4.460 \\
\hline Occupational therapy & & & & & \\
Age & -0.017 & 0.016 & 0.291 & 0.983 & 0.951 to 1.014 \\
\hline Post-stroke duration (day) & -0.016 & 0.009 & 0.091 & 0.984 & 0.966 to 1.001 \\
\hline Stroke etiology & 0.242 & 0.448 & 0.589 & 1.274 & 0.396 to 2.152 \\
Stroke recurrence & -0.895 & 1.295 & 0.490 & 0.409 & -2.129 to 2.947 \\
\hline Laterality & -0.181 & 0.389 & 0.642 & 0.835 & 0.073 to 1.597 \\
\hline Location & 1.293 & 0.849 & 0.128 & 3.644 & 1.965 to 5.323 \\
\hline MBI & 0.011 & 0.010 & 0.252 & 1.011 & 0.991 to 1.031 \\
\hline
\end{tabular}

Goal attainment was assessed as having a standard GAS score $\geq 50$ in each department (physical therapy or occupational therapy). Etiology of stroke was classified into cerebral infarction and intracerebral hemorrhage. The stroke laterality was classified as right, left and both sides. The table shows the OR of the left side for the right side. Stroke location was classified as cerebral hemisphere lesion and brainstem or cerebellar lesion.

B, regression coefficient; SE, standard error; OR, odds ratio; CI, confidence interval; MBI, Modified Barthel Index; GAS, goal attainment scaling.

${ }^{*} \mathrm{p}<0.05$. 
Table 5. Comparison between the group with MMSE $<20$ and the group with MMSE $\geq 20$

\begin{tabular}{|c|c|c|c|}
\hline & Group with MMSE $<20(n=85)$ & Group with MMSE $\geq 20(n=56)$ & p-value \\
\hline Age (yr) & $64.0 \pm 12.9$ & $54.0 \pm 13.5$ & $<0.01^{* *}$ \\
\hline Sex & & & 0.10 \\
\hline Male & 46 & 38 & \\
\hline Female & 39 & 18 & \\
\hline Etiology & & & 0.06 \\
\hline Cerebral infarction & $30(35.3)$ & $29(51.8)$ & \\
\hline Intracerebral hemorrhage & $55(64.7)$ & $27(48.2)$ & \\
\hline Lesion & & & $0.04^{*}$ \\
\hline Right hemisphere & $37(43.5)$ & $32(57.1)$ & \\
\hline Left hemisphere & $35(41.2)$ & $19(33.9)$ & \\
\hline Bilateral hemispheres & $3(3.5)$ & $5(9.0)$ & \\
\hline Cerebellum & $2(2.4)$ & $0(0.0)$ & \\
\hline Brainstem & $8(9.4)$ & $0(0.0)$ & \\
\hline Post-stroke duration (day) & $40.4 \pm 25.2$ & $25.5 \pm 12.9$ & $<0.01^{* *}$ \\
\hline Recurred stroke & $2(2.4)$ & $1(1.8)$ & 0.82 \\
\hline MMSE score & $10.2 \pm 6.9$ & $23.7 \pm 2.8$ & $<0.01^{* *}$ \\
\hline FMA score & $26.2 \pm 22.9$ & $38.4 \pm 24.4$ & $<0.01^{* *}$ \\
\hline MFT score & $28.9 \pm 31.7$ & $47.7 \pm 34.8$ & $<0.01^{* *}$ \\
\hline MBI score & $26.3 \pm 19.9$ & $50.8 \pm 22.2$ & $<0.01^{* *}$ \\
\hline \multicolumn{4}{|l|}{ FIM score } \\
\hline Cognition & $14.0 \pm 6.4$ & $26.2 \pm 5.1$ & $<0.01^{* *}$ \\
\hline Motor & $28.9 \pm 13.2$ & $48.0 \pm 17.2$ & $<0.01^{* *}$ \\
\hline MMT score & $11.0 \pm 5.3$ & $14.0 \pm 4.8$ & $<0.01^{* *}$ \\
\hline BBS score & $13.2 \pm 16.0$ & $24.6 \pm 19.7$ & $<0.01^{* *}$ \\
\hline TIS score & $6.27 \pm 6.18$ & $11.50 \pm 6.04$ & $<0.01^{* *}$ \\
\hline FAC & $0.91 \pm 1.03$ & $1.80 \pm 1.44$ & $<0.01^{* *}$ \\
\hline \multicolumn{4}{|l|}{ Standard GAS score } \\
\hline Physical therapy & $49.1 \pm 8.0$ & $54.3 \pm 6.8$ & $<0.01^{* *}$ \\
\hline Occupational therapy & $47.1 \pm 6.9$ & $49.0 \pm 6.4$ & $0.04^{*}$ \\
\hline
\end{tabular}

Values are presented as mean \pm standard deviation or number (\%).

The sum of the manual muscle test by Medical Research Council score was divided into 6 muscle groups including shoulder abductor of the weaker side, elbow flexor, wrist extensor, hip flexor, knee extensor, and ankle dorsiflexor. It ranges from 0 (totally hemiplegic) to 30 (normal strength).

MMSE, Mini-Mental State Examination; FMA, Fugl-Meyer Assessment; MFT, Manual Function Test; MBI, Modified Barthel Index; FIM, Functional Independence Measurement; MMT, Manual Muscle Test; BBS, Berg Balance Scale; TIS, Trunk Impairment Scale; FAC, Functional Ambulatory Category; GAS, goal attainment scaling.

Number of each group was compared by chi-square test. Other characteristics and evaluation scores were compared by Mann-Whitney U-test.

${ }^{*} \mathrm{p}<0.05,{ }^{* *} \mathrm{p}<0.01$.

the introduction of GAS, therapists thought that patients were more likely to participate in their treatment. They also thought that the goal setting and healing process improved the quality of the treatment and thought that GAS was a good way to evaluate treatment results (Table 6).

\section{DISCUSSION}

GAS appears to be useful in the intensive rehabilitation of patients after a subacute stroke based on the above results. There were three meaningful findings in this 

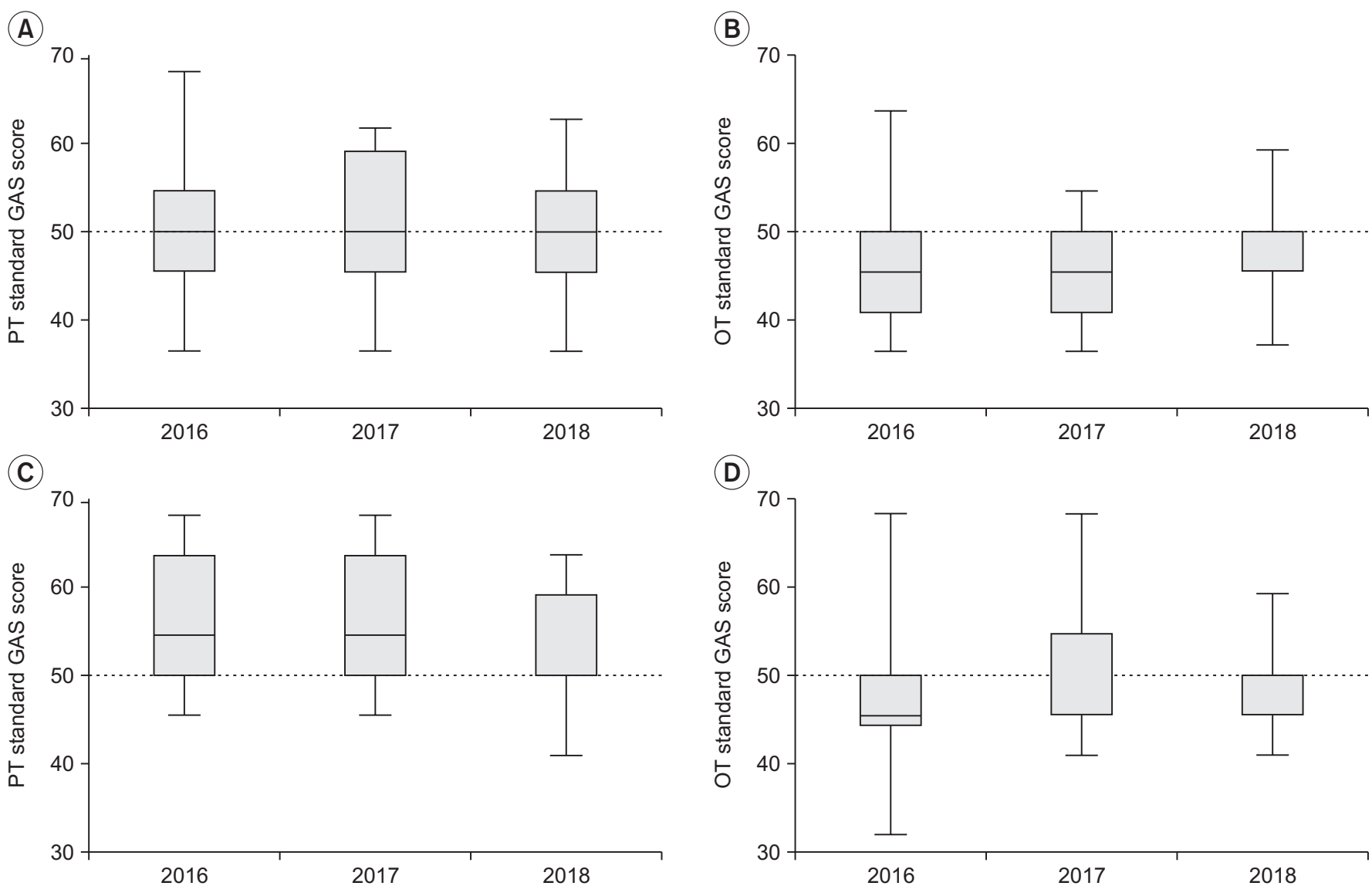

Fig. 2. Changes in standard goal attainment scaling (GAS) scores during 3 years classified by cognitive ability. (A) PT domain and (B) OT domain in the low condition group (MMSE <20). (C) PT domain and (D) OT domain in the high condition group (MMSE $\geq 20$ ). The box-and-whiskers plots represent line is median value; box, upper and lower quartiles; whiskers, 5\%-95\% confidence. The median values are displayed above the box. MMSE, Mini-Mental State Examination.

Table 6. Distribution of questionnaire results with five options for therapists ( $\mathrm{n}=13)$

\begin{tabular}{|c|c|c|c|c|c|}
\hline \multirow[b]{2}{*}{ Question } & \multicolumn{5}{|c|}{ Response (\%) } \\
\hline & $\begin{array}{c}\text { Strongly } \\
\text { agree }\end{array}$ & Agree & $\begin{array}{l}\text { Neither agree/ } \\
\text { nor disagree }\end{array}$ & Disagree & $\begin{array}{l}\text { Strongly } \\
\text { disagree }\end{array}$ \\
\hline $\begin{array}{l}\text { The GAS is a good way to evaluate treatment } \\
\text { results. }\end{array}$ & 7.7 & 76.9 & 15.4 & 0 & 0 \\
\hline $\begin{array}{l}\text { Application of GAS facilitated setting specific } \\
\text { goals. }\end{array}$ & 7.7 & 76.9 & 15.4 & 0 & 0 \\
\hline $\begin{array}{l}\text { Use of the GAS improves quality of the } \\
\text { rehabilitation treatment. }\end{array}$ & 15.4 & 69.2 & 15.4 & 0 & 0 \\
\hline $\begin{array}{l}\text { By using GAS, patients can participate with a } \\
\text { good understanding of their treatment } \\
\text { process. }\end{array}$ & 7.7 & 61.5 & 23.1 & 7.7 & 0 \\
\hline $\begin{array}{l}\text { Use of the GAS enhances motivation of the } \\
\text { patient. }\end{array}$ & 0.0 & 69.2 & 23.1 & 7.7 & 0 \\
\hline
\end{tabular}

GAS, goal attainment scaling. 
study. First, the standard GAS scores in both the PT and OT domains gradually reached 50 over the 3-year period. Initially, the PT domain scores were high, and the OT domain scores were low. This signals an improvement in GAS quality in both domains due to experience [10]. Second, the logistic regression analysis of factors that were expected to affect the standard GAS score showed that only the post-stroke duration had a significantly negative impact. Based on these findings, we can set the goal in consideration of the post-stroke duration at the beginning of rehabilitation. Finally, a survey of therapists implies that GAS would be beneficial to the rehabilitation process.

The subacute period in stroke patients is the time when many functional improvements tend to take place. These improvements vary from patient to patient [2]. Therefore, in rehabilitation practice, it can be challenging to set the goals appropriately, especially for inexperienced doctors and therapists [20]. The rehabilitation team in the study hospital had a weekly meeting led by a physiatrist and supported by an experienced therapist about the goal setting for new patients and GAS assessment of the patients who had completed rehabilitation during admission. In this process, various individual personal factors of the patient (age, initial neurological impairment status, and medical conditions) were considered, and the physiatrist guided the adjustment of the goals according to that information. In addition, GAS scores of patients who completed rehabilitation therapy were evaluated. As a result of using GAS, both the PT and OT domains accurately predicted the patient's expected improvement.

Goal attainment scaling is a tool for assessing the achievement of a client-centered goal and can evaluate the effectiveness of the intervention at multiple levels [10]. GAS allows the achievement of different goals to be assessed for each patient. Additionally, the GAS score reflects the relative importance or difficulty of the goal [21]. In this study, three goals were selected for the PT and OT domains, and the achievement of each goal was evaluated in an integrated manner as a standard GAS score. GAS scores were higher than 50 in the PT domain and lower than 50 in the OT domain. This means that the patients' improvement was higher than the goal in the PT domain and lower than the goal in the OT domain [10]. In detail, the PT domain was seen to have high standard GAS scores in muscle function (b730-b749), walking and moving (d450-d469), and the OT showed low standard GAS scores in carrying, moving and handling objects (d430-d449). The upper functional recovery of the upper extremities is low, with $5 \%-34 \%$ complete functional recovery of the upper extremities up to 6 months after onset in stroke patients [22]. On the other hand, gait is known to recover in more patients, at a rate of about $70 \%$ [23]. The differences between upper and lower limb recovery may have influenced the differences seen between the two domains. Over time, as goal-setting became more appropriate, feedback on the GAS scores would have helped.

The post-stroke duration at the time of the initial evaluation was found to be a factor that affected goal achievement. The longer the post-stroke duration at admission to the rehabilitation center was, the lower the probability of achieving the goal. Therefore, post-stroke duration should be considered when setting goals regarding the patient's functional gain after stroke. This is consistent with previous Cochrane review findings that the shorter the post-stroke duration before rehabilitation, the better the functional improvement [24]. Other characteristics, such as the initial ADL score, which reflect the severity and location of the stroke, did not correlate with goal achievement. Additionally, we examined the changes in the standard GAS scores by dividing those with good and poor cognition into two groups according to baseline MMSE score. There was no significant trend identified except that higher cognitive groups had higher standard GAS scores in both the PT and OT domains. Therefore, it appears that the higher the patient's cognition is, the better the functional performance achieved [10]. This is consistent with a previous report that the cognitive function level at admission is related to goal attainment in inpatient neurorehabilitation [25]. This may be because stroke patients with poor cognition showed poor participation in rehabilitation due to problems such as poor understanding of instructions or inability to perform self-directed rehabilitation regimens [26]. However, the fact that the post-stroke duration, identified as a factor influencing goal attainment in our study, was shorter in the high cognition level group than in the low cognition group does limit the interpretation of this result.

From the survey undertaken by the rehabilitation team members, we found that they thought that GAS improved treatment quality and was helpful for goal setting. Ac- 
cording to a review paper, setting goals that reproduce one's specific rehabilitation target may improve the results of the intervention [2]. It can be inferred that GAS may be helpful for treatment. The therapists also felt that patients appeared to be more motivated. Previous literature has reported that GAS improves the patient's motivation and the treatment effect $[27,28]$. In particular, the patient and their family can have an accurate understanding of the prognosis and, thus, can find a useful role in real life [29]. However, this study was retrospective, so we could not directly confirm whether the patient's motivation levels did improve.

There are some challenges with using GAS. It takes time to be proficient in using GAS, particularly in terms of goal setting and assessing GAS $[20,30]$. Furthermore, it is pointed out that the discontinuity $(-2,-1,0,+1$, and +2$)$ that assesses whether an expected outcome is achieved or not is not appropriate in assessing partial goal attainment [31]. Finally, GAS alone does not reflect the patient's absolute function [32]. However, despite these drawbacks, GAS has been shown to be an appropriate outcome assessment tool in adult physical and neurological rehabilitation settings [9].

This study had several limitations. This retrospective analysis did not evaluate the motivation levels of the patients and their caregivers. Also, it should be noted that different results can be obtained in teams that are not accustomed to using GAS, or that are less experienced in the rehabilitation of post-stroke patients in the subacute period. A larger prospective study at multiple centers may help to overcome these limitations. Finally, in some cases, the criteria between GAS 1 and 2 were only partially predefined in the initial team meeting, and thus in many cases, were subjectively determined when the final GAS score was given.

In conclusion, by introducing GAS, we found that the rehabilitation medicine team showed gradual improvement in appropriate goal setting for post-stroke patients in the subacute period. We also found that GAS may have positive effects on the patient's treatment. The degree of goal achievement can be influenced by the post-stroke duration at the beginning of rehabilitation. Introducing GAS in the rehabilitation of post-stroke patients in the subacute period may be useful and may be helpful for rehabilitation therapy.

\section{CONFLICT OF INTEREST}

No potential conflict of interest relevant to this article was reported.

\section{ACKNOWLEDGMENTS}

I would like to thank all the therapists who participated in the survey. I would also like to thank Jee-Il Kim from the University of Southern California for his English editing.

This research was supported by a grant from the Korea Health Technology R\&D Project through the Korea Health Industry Development Institute (KHIDI), funded by the Ministry of Health \& Welfare, Republic of Korea (No. HI16C1559).

\section{AUTHOR CONTRIBUTION}

Conceptualization: Kim MY, Jung YS. Methodology: Kim MY, Jung YS, Kim JM. Formal analysis: Kim MY, Jung YS, Sim JH, Park JH. Project administration: Kim MY, Jung YS. Visualization: Jung YS, Sim JH, Park JH, Kim JM. Writing - original draft: Kim MY, Jung YS. Writing - review and editing: Kim MY, Jung YS. Approval of the final manuscript: all authors.

\section{SUPPLEMENTARY MATERIALS}

Supplementary Materials can be found via https://doi. org/10.5535/arm.19087.

\section{REFERENCES}

1. Bonita R, Mendis S, Truelsen T, Bogousslavsky J, Toole J, Yatsu F. The global stroke initiative. Lancet Neurol 2004;3:391-3.

2. Langhorne P, Bernhardt J, Kwakkel G. Stroke rehabilitation. Lancet 2011;377:1693-702.

3. Carod-Artal FJ, Gonzalez-Gutierrez JL, Herrero JA, Horan T, De Seijas EV. Functional recovery and instrumental activities of daily living: follow-up 1-year after treatment in a stroke unit. Brain Inj 2002;16:207-16.

4. Dobkin BH. Clinical practice: rehabilitation after stroke. N Engl J Med 2005;352:1677-84.

5. Wressle E, Oberg B, Henriksson C. The rehabilitation 
process for the geriatric stroke patient: an exploratory study of goal setting and interventions. Disabil Rehabil 1999;21:80-7.

6. Siegert RJ, Taylor WJ. Theoretical aspects of goal-setting and motivation in rehabilitation. Disabil Rehabil 2004;26:1-8.

7. Sugavanam T, Mead G, Bulley C, Donaghy M, van Wijck F. The effects and experiences of goal setting in stroke rehabilitation: a systematic review. Disabil Rehabil 2013;35:177-90.

8. Kiresuk TJ, Sherman RE. Goal attainment scaling: a general method for evaluating comprehensive community mental health programs. Community Ment Health J 1968;4:443-53.

9. Hurn J, Kneebone I, Cropley M. Goal setting as an outcome measure: a systematic review. Clin Rehabil 2006;20:756-72.

10. Turner-Stokes L. Goal attainment scaling (GAS) in rehabilitation: a practical guide. Clin Rehabil 2009;23:362-70.

11. Krasny-Pacini A, Hiebel J, Pauly F, Godon S, Chevignard M. Goal attainment scaling in rehabilitation: a literature-based update. Ann Phys Rehabil Med 2013;56:212-30.

12. Grant M, Ponsford J. Goal attainment scaling in brain injury rehabilitation: strengths, limitations and recommendations for future applications. Neuropsychol Rehabil 2014;24:661-77.

13. Malec JF, Smigielski JS, DePompolo RW. Goal attainment scaling and outcome measurement in postacute brain injury rehabilitation. Arch Phys Med Rehabil 1991;72:138-43.

14. Brock K, Black S, Cotton S, Kennedy G, Wilson S, Sutton E. Goal achievement in the six months after inpatient rehabilitation for stroke. Disabil Rehabil 2009;31:880-6.

15. Bernhardt J, Dewey H, Thrift A, Donnan G. Inactive and alone: physical activity within the first 14 days of acute stroke unit care. Stroke 2004;35:1005-9.

16. Lim KB, Lee HJ, Yoo J, Kwon YG. Effect of lowfrequency rTMS and NMES on subacute unilateral hemispheric stroke with dysphagia. Ann Rehabil Med 2014;38:592-602.

17. Jimenez Bunuales MT, Gonzalez Diego P, Martin Moreno JM. International Classification of Functioning, Disability and Health (ICF) 2001. Rev Esp Salud
Publica 2002;76:271-9.

18. Barbarotto R, Cerri M, Acerbi C, Molinari S, Capitani E. Is SIB or BNP better than MMSE in discriminating the cognitive performance of severely impaired elderly patients? Arch Clin Neuropsychol 2000;15:21-9.

19. Bucher L. Evaluating the affective domain: consider a Likert scale. J Nurs Staff Dev 1991;7:234-8.

20. Stolee P, Awad M, Byrne K, Deforge R, Clements S, Glenny C, et al. A multi-site study of the feasibility and clinical utility of Goal Attainment Scaling in geriatric day hospitals. Disabil Rehabil 2012;34:1716-26.

21. Steenbeek D, Ketelaar M, Galama K, Gorter JW. Goal attainment scaling in paediatric rehabilitation: a critical review of the literature. Dev Med Child Neurol 2007;49:550-6.

22. Kong KH, Chua KS, Lee J. Recovery of upper limb dexterity in patients more than 1 year after stroke: frequency, clinical correlates and predictors. NeuroRehabilitation 2011;28:105-11.

23. Buma F, Kwakkel G, Ramsey N. Understanding upper limb recovery after stroke. Restor Neurol Neurosci 2013;31:707-22.

24. Pollock A, Baer G, Campbell P, Choo PL, Forster A, Morris J, et al. Physical rehabilitation approaches for the recovery of function and mobility following stroke. Cochrane Database Syst Rev 2014;(4):CD001920.

25. Prigatano GP, Wong JL. Cognitive and affective improvement in brain dysfunctional patients who achieve inpatient rehabilitation goals. Arch Phys Med Rehabil 1999;80:77-84.

26. Skidmore ER, Whyte EM, Holm MB, Becker JT, Butters MA, Dew MA, et al. Cognitive and affective predictors of rehabilitation participation after stroke. Arch Phys Med Rehabil 2010;91:203-7.

27. Wressle E, Eeg-Olofsson AM, Marcusson J, Henriksson C. Improved client participation in the rehabilitation process using a client-centred goal formulation structure. J Rehabil Med 2002;34:5-11.

28. Wade DT. Goal setting in rehabilitation: an overview of what, why and how. Clin Rehabil 2009;23:291-5.

29. Bouwens SF, van Heugten CM, Verhey FR. The practical use of goal attainment scaling for people with acquired brain injury who receive cognitive rehabilitation. Clin Rehabil 2009;23:310-20.

30. Doig E, Fleming J, Kuipers P, Cornwell PL. Clinical utility of the combined use of the Canadian Occupa- 
Youngsu Jung, et al.

tional Performance Measure and Goal Attainment Scaling. Am J Occup Ther 2010;64:904-14.

31. Bovend'Eerdt TJ, Botell RE, Wade DT. Writing SMART rehabilitation goals and achieving goal attainment scaling: a practical guide. Clin Rehabil 2009;23:352-
61.

32. Forbes DA. Goal Attainment Scaling: a responsive measure of client outcomes. J Gerontol Nurs 1998;24:34-40. 